\title{
Fingerprinting fission yeast: polymorphic markers for molecular genetic analysis of Schizosaccharomyces pombe strains
}

\section{Correspondence \\ Stephen Aves \\ S.J.Aves@exeter.ac.uk}

Received 23 August 2006

Revised 9 November 2006

Accepted 20 November 2006

\author{
Ann-Marie Patch and Stephen J. Aves
}

\author{
School of Biosciences, University of Exeter, Geoffrey Pope Building, Stocker Road, Exeter \\ EX4 4QD, UK
}

\begin{abstract}
The fission yeast Schizosaccharomyces pombe is widely used as a model eukaryote for cell and molecular studies but little is known of natural genetic variation in this species. In order to obtain informative molecular markers, imperfect tandem repeats, identified through bioinformatic methods, were tested for length polymorphism in six wild-type strains of Sch. pombe isolated from different substrates and geographical locations in Africa, America, Asia and Europe. Of 26 loci tested, 21 were multi-allelic, consistent with tandem repeat copy number variation. Eleven of these polymorphic tandem repeats are in regions encoding intracellular proteins. Most of the protein-coding repeats are not sited within structured domains but have non-regular predicted structure; one has a repeat unit length corresponding to integer turns of a predicted amphipathic $\alpha$-helix secondary structure, suggesting that this repeat may be tolerated because copy number mutations change $\alpha$-helix length but not orientation within the protein structure. In contrast to the differences observed between natural isolates of Sch. pombe, genetic strains were found to be essentially isogenic: only two polymorphic loci were detected out of 26 minisatellites and five microsatellites tested in 16 strains, including a hypervariable microsatellite in the med15 gene. The polymorphic tandem repeat markers identified in this study will prove useful for DNA fingerprinting and molecular analysis of natural genetic variation in Sch. pombe isolates.
\end{abstract}

\section{INTRODUCTION}

The fission yeast Schizosaccharomyces pombe has been widely exploited as a model eukaryote for genetic, cell and molecular biological studies, particularly relating to mating type and the cell cycle (Egel, 2004). Sch. pombe was one of the first eukaryotes to have its genome completely sequenced and annotated (Wood et al., 2002) and it has fully entered the post-genomic age, with a wide range of molecular genetic, genomic and functional genomic techniques available to study its genome and its proteome, which at 4912 predicted proteins is one of the smallest for a free-living eukaryote (Sunnerhagen, 2002; Forsburg \& Rhind, 2006; Marguerat et al., 2006). Sch. pombe and two other fission yeast species of the same genus form a distinct group of ascomycete fungi which are phenotypically and phylogenetically highly divergent from budding yeasts such as Saccharomyces cerevisiae or Candida albicans (Kurtzman \& Robnett, 1998; Sipiczki, 2000). Differing estimates put the

Abbreviations: CBS, Centraalbureau voor Schimmelcultures; CTD, Cterminal domain; indels, insertions or deletions; NCYC, National Collection of Yeast Cultures.

Three supplementary figures are available with the online version of this paper. divergence of Sch. pombe and Sac. cerevisiae at 330-420 or 1070-1220 million years ago and, when compared with calculations for the divergence of fungi and metazoa at between 1000-1100 or 1600 million years ago, indicate a long evolutionary separation (Sipiczki, 2000; Heckman et al., 2001). This large evolutionary divergence between fission and budding yeasts makes Sch. pombe a particularly valuable model organism alongside Sac. cerevisiae, as comparisons between these species can be very informative for extrapolation to other eukaryotes (Forsburg, 1999).

As a model organism, Sch. pombe has the great advantage that all genetic strains and mutants derive from a single isolate, and are therefore assumed to be isogenic. Genetic strains were derived by Leupold (1950) from a Sch. pombe strain isolated in 1924 from grape juice originating from Southern France, and originally designated Sch. liquefaciens (Osterwalder, 1924). This Sch. pombe wild-type strain is just one of many dozens isolated from substrates ranging from fruit juice and molasses to fermented beverages from all inhabited continents of the world, particularly in the decades following the original description of this species isolated from East African millet beer (the word 'pombe' is Swahili for beer; Lindner, 1893). In contrast to the concentration of research on genetic strains of Sch. pombe, 
relatively few studies have been carried out on natural variants of this fission yeast species, and very few indeed using molecular genetic techniques. Study and sequencing of budding yeasts, such as the Saccharomyces sensu stricto group, has demonstrated the valuable insights that can be gained from the molecular analysis of natural variation in yeasts (Kellis et al., 2003).

The variability of tandem repeats has led to their widespread use as genetic markers for mapping, population and DNA fingerprinting studies (Calafell et al., 1998). Tandem repeats are approximate or exact head-to-tail copies of a nucleotide sequence and are often classified by repeat unit size as microsatellites or minisatellites. They occur in both proteincoding and intergenic regions of eukaryotic genomes from yeast to humans (Vergnaud \& Denoeud, 2000; Tóth et al., 2000; Katti et al., 2001) and there is accumulating evidence that they may serve functional roles within coding sequences and as regulatory elements or mutational hotspots (Kashi et al., 1997; Fondon \& Garner, 2004; Verstrepen et al., 2005; Bowen \& Wheals, 2006). Tandem repeats frequently exhibit copy number polymorphisms caused by mechanisms such as DNA replication slippage and asymmetric recombination (Debrauwère et al., 1997; Chambers \& MacAvoy, 2000; Richard \& Pâques, 2000; Viguera et al., 2001) and in humans, expansions of microsatellites (tandem repeats with a short repeat unit length) have been associated with genetic diseases including neurodegenerative disorders such as fragile X syndrome and Huntington's disease (Sutherland \& Richards, 1995; Mitas, 1997; Tóth et al., 2000; Boby et al., 2005).

In this study we screened the Sch. pombe genome for tandem repeats that can be used as markers for genetically fingerprinting this species of fission yeast. We demonstrate the usefulness of these markers for exploring the similarities and differences between Sch. pombe isolates from different geographical locations, and we investigate the extent of tandem repeat polymorphism within genetic strains of Sch. pombe.

\section{METHODS}

Strains. Sch. pombe wild-type strains representing different geographical isolates were obtained from the Centraalbureau voor Schimmelcultures (CBS), Utrecht, The Netherlands, and two control Leupold (1950) strains were obtained from the National Collection of Yeast Cultures (NCYC), Norwich, UK. All isolates were sequenced at the rDNA ITS locus to confirm that they were strains of Sch. pombe. Genetic strains were obtained from the Sch. pombe community. Details of all strains are shown in Table 1.

Selection of tandem repeat markers and primer design. Potential fingerprinting targets were selected from a comprehensive analysis of the whole Sch. pombe genome sequence (Wood et al., 2002) for perfect and approximate tandem repeats, performed using Tandem Repeats Finder (Benson, 1999; A.-M. Patch, \& S. J. Aves unpublished). Minisatellite and microsatellite tandem repeats were selected as candidates for fingerprinting from protein-coding and non-coding regions using the following criteria: $\geqslant 60 \%$ matches of repeat sequences to the consensus, $\leqslant 16 \%$ insertions or deletions (indels), an overall estimated fragment length between 175 and $600 \mathrm{bp}$, and, where possible, $\geqslant 4$ repeated copies. Minisatellites were defined as having repeat units of $\geqslant 7 \mathrm{bp}$. These criteria were chosen to provide tandem repeat alleles that could potentially be resolved by agarose gel electrophoresis. Excluded from this group were any tandem repeats that crossed exon boundaries ( $<2 \%$ of total).

Primer sequences were selected from sequences flanking the target tandem repeats and separated by at least $20 \mathrm{nt}$ each side. Optimal primers were designed using the web-accessible version of Primer3 (Rozen \& Skaletsky, 2000) with ideal $T_{\mathrm{m}}$ set at $57^{\circ} \mathrm{C}$, ideal primer length of $21 \mathrm{bp}$, and other default parameters. All primer pairs were assessed for primer-primer interactions using the web-accessible 'Net Primer' program (http://www.premierbiosoft.com/netprimer/ index.html). Oligonucleotide primers were synthesized by MWG Biotech and details of these are given in Table 2.

DNA preparation and PCR amplification. Sch. pombe strains were cultured in YE medium and DNA was extracted according to the protocol of Moreno et al. (1991) but using $2 \mathrm{mg}$ lyticase $\mathrm{ml}^{-1}$ (Sigma-Aldrich) in place of Zymolyase-20T for cell lysis. PCR reactions were in $50 \mu \mathrm{l}$ containing $30 \mathrm{ng}$ DNA, $1.5 \mathrm{mM} \mathrm{MgCl}_{2}, 200 \mu \mathrm{M}$ of each dNTP, $20 \mathrm{pmol}$ of each primer, 1 unit Taq polymerase and $1 \times$ Taq buffer (Promega or New England Biolabs). PCR reactions had an initial denaturation step of $2 \mathrm{~min}$ at $94^{\circ} \mathrm{C}$ followed by 30 cycles of $20 \mathrm{~s}$ denaturation at $94{ }^{\circ} \mathrm{C}, 30 \mathrm{~s}$ annealing at $57^{\circ} \mathrm{C}$ and $1 \mathrm{~min} 15 \mathrm{~s}$ elongation at $72^{\circ} \mathrm{C}$; there was a final extension step at $72{ }^{\circ} \mathrm{C}$ for $5 \mathrm{~min}$. PCR products were fractionated in $2 \%$ agarose TBE gels alongside $100 \mathrm{bp}$ ladder (New England Biolabs).

Sequencing and data analysis. PCR products were sequenced directly after cleaning by ethanol precipitation; $2 \mathrm{ng}$ template was used in a $10 \mu \mathrm{l}$ reaction also containing $2 \mu \mathrm{l}$ BigDye Terminator (Applied Biosystems), $2 \mu \mathrm{l} \mathrm{HalfTerm} \mathrm{buffer} \mathrm{(Genpak),} \mathrm{and} 10 \mathrm{pmol}$ primer. Reactions had an initial denaturing step of $1 \mathrm{~min}$ at $70^{\circ} \mathrm{C}$ followed by 25 cycles of $10 \mathrm{~s}$ denaturation at $96^{\circ} \mathrm{C}, 5 \mathrm{~s}$ annealing at $50{ }^{\circ} \mathrm{C}, 4 \mathrm{~min}$ elongation at $60^{\circ} \mathrm{C}$, and a final step at $60^{\circ} \mathrm{C}$ for $5 \mathrm{~min}$. Products were ethanol precipitated and sequenced on an ABI3100 automated sequencer. Electropherograms were analysed using the freeware version of CHROMAS (Technelysium) and compared with the genomic template using BLASTN. Sequence alignments were produced with CLUSTALW (Thompson et al., 1994). Protein structure prediction was carried out using the web-accessible software PredictProtein (Rost et al., 2003) and PSIPRED (McGuffin et al., 2000). Predictions of intrinsically unstructured/disordered protein domains were confirmed by two independent disorder prediction software tools DISOPRED2 and vsL2 (Ward et al., 2004; Peng et al., 2006).

A phenetic unrooted dendrogram was constructed, based on a distance matrix obtained by counting the number of differences between corresponding alleles, using neighbour-joining cluster analysis performed with PHYLIP (Phylogeny Inference Package) version 3.6 (Felsenstein, 2005).

\section{RESULTS}

\section{Minisatellite markers enable DNA fingerprinting of Sch. pombe isolates}

In order to determine informative markers for DNA fingerprinting of Sch. pombe strains, a subset of tandem repeats identified bioinformatically in the sequenced Sch. pombe genome was selected (see Methods). The screening criteria were devised so that polymorphisms could be readily identified using standard agarose gels, ensuring that total amplicon lengths exceeded $175 \mathrm{bp}$. High internal conserva- 
Table 1. Sch. pombe strains

\begin{tabular}{|c|c|c|c|c|c|}
\hline Strain ${ }^{\star}$ & Genotype & Obtained from $\dagger$ & Original isolate & Substrate & Country of origin \\
\hline 972 & $h^{-}$ & NCYC 1827 & $\begin{array}{l}\text { Osterwalder (formerly } \\
\text { Sch. liquefaciens) }\end{array}$ & Grape juice & France \\
\hline CBS356 & Wild-type & CBS & Sch. pombe Lindner type strain & & \\
\hline CBS2775 & Wild-type & CBS & $\begin{array}{l}\text { K. Sakaguchi (formerly Sch. } \\
\text { pombe var. ogasawaraensis) }\end{array}$ & Fermenting molasses & Japan \\
\hline CBS5557 & Wild-type & CBS & $\begin{array}{l}\text { Rankine \& Fornachon } \\
\text { (formerly Sch. malidevorans) }\end{array}$ & Listan grapes & Spain \\
\hline CBS5682 & Wild-type & CBS & J. P. van der Walt & Sorghum beer & $\begin{array}{l}\text { Swaziland, } \\
\text { Southern Africa }\end{array}$ \\
\hline SJA216 & $h^{-}$nda4-108 leu1-32 & M. Yanagida & Osterwalder & Grape juice & France \\
\hline SJA237 & $h^{-}$cut5-580 leu1-32 & M. Yanagida & Osterwalder & Grape juice & France \\
\hline SJA273 & $h^{-}$nda3-KM311 & M. Yanagida & Osterwalder & Grape juice & France \\
\hline SJA277 & $h^{-} c d c 23-M 30$ & P. Nurse & Osterwalder & Grape juice & France \\
\hline SJA272 & $h^{-} c d c 25-22$ & P. Nurse & Osterwalder & Grape juice & France \\
\hline SJA244 & $\begin{array}{l}h^{-} c d c 23-I E 2 \\
\text { ade6-704 leu1-32 }\end{array}$ & P. Nurse & Osterwalder & Grape juice & France \\
\hline SJA122 & $\begin{array}{l}h^{-} \text {ura4-294 tps14-5 } \\
\text { ade5-36 swi5-39 }\end{array}$ & H. Schmidt & Osterwalder & Grape juice & France \\
\hline SJA120 & $h^{90}$ swi5-39 & H. Schmidt & Osterwalder & Grape juice & France \\
\hline SJA238 & $h^{-}$ade6-M210 & H. Schmidt & Osterwalder & Grape juice & France \\
\hline
\end{tabular}

${ }^{*}$ Strains 972 and 975 are from Leupold (1950); genetic strains are identified by an SJA prefix.

$\dagger$ CBS, Centraalbureau voor Schimmelcultures, The Netherlands; NCYC, National Collection of Yeast Cultures, Norwich, UK; R. Egel, Institute of Molecular Biology, University of Copenhagen, Denmark; P. Thuriaux, CEA/Saclay, Gif-sur-Yvette, France; M. Yanagida, Kyoto University, Japan; P. Nurse, Rockefeller University, New York, USA; H. Schmidt, Institut für Genetik, Technische Universität Braunschweig, Germany; A. Carr, Genome Damage and Stability Centre, University of Sussex, UK; S. Forsburg, University of Southern California, USA.

tion of sequence between repeated units increases the likelihood of polymorphic alleles (Strand et al., 1993); therefore a minimum value of $60 \%$ was set for mean percentage identity between repeat copies, and a maximum value of $16 \%$ for indels (insertions and deletions); note that all of the selected tandem repeats were imperfect. Tandem repeat targets were selected from both protein-coding and non-coding regions of the genome to allow comparison of tandem repeat polymorphism rates between these. In total 26 minisatellites (Table 2) were chosen which best matched the screening criteria: 15 within protein-coding sequences and 11 within intergenes. Flanking primers were designed for PCR amplification of each of these, ensuring that they were not sited within any residual degenerate copies of the repeats.
The usefulness of the 26 minisatellites as genetic markers was tested on six independently identified Sch. pombe isolates. These were chosen to include widespread geographical origins in Africa, Asia, Europe and America, and to represent a diversity of source substrates including molasses, fruit juice and alcoholic beverages (Table 1). The two heterothallic strains $972 h^{-}$and $975 h^{+}$(Leupold, 1950) were representatives of the Osterwalder (1924) isolate from French grape juice which has given rise to the genetic strains used in virtually all molecular studies on Sch. pombe. The other five chosen isolates have been the subject of comparative molecular studies: CBS5557, formerly known as Sch. malidevorans, has been used for analysis of tandem repeats in the cpy1 gene (Ingavale et al., 1998) and, together with the Sch. pombe type strain CBS356 (also known as EF1), 
Table 2. Tandem repeat characteristics and primer sequences

\begin{tabular}{|c|c|c|c|c|c|c|c|}
\hline No. & Position $^{\star}$ & $\begin{array}{l}\text { Repeat unit } \\
\text { length } \dagger\end{array}$ & Copy no. & $\begin{array}{l}\text { Fragment } \\
\text { length } \S\end{array}$ & Match (\%) & Primer sequences & AllelesII \\
\hline 1 & SPAC13G6.10c & 33 & 4.1 & 206 & 71 & $\begin{array}{l}\text { L: GACAGGAGCCACATAGGATGA } \\
\text { R: CCTGTATCAACTCCCGAGACA }\end{array}$ & 1 \\
\hline 2 & spt5 & 9 & 5.2 & 241 & 84 & $\begin{array}{l}\text { L: AACAGAGTTCTCCCGTGGATT } \\
\text { R: CTTTTTCTTCTACCGCCACCT }\end{array}$ & 1 \\
\hline 3 & SPAC56F8.12-SPAC56F8.14c & 9 & 12.7 & 384 & 71 & $\begin{array}{l}\text { L: CAATAGAATCGGGTGAGGTACG } \\
\text { R: AATGCTGCTGGTAAAGATATGC }\end{array}$ & 1 \\
\hline 4 & gar2 & 21 & 4.0 & 229 & 76 & $\begin{array}{l}\text { L: AGTCCCGAACCTTCAAAGAAA } \\
\text { R: CGCTGGAACTCTCCTTCTTCT }\end{array}$ & 3 \\
\hline 5 & SPAC16E8.01 & 21 & 4.4 & 466 & 91 & $\begin{array}{l}\text { L: GCCACTGGTTATCAACCTTTA } \\
\text { R: GAACTTGCACACCAGTCATCT }\end{array}$ & 2 \\
\hline 6 & cpyl & 27 & 7.6 & 293 & 93 & $\begin{array}{l}\text { L: GAGAACTTTGCATGTTTTGCTC } \\
\text { R: TCCTTTCAAACACCATGAGCTT }\end{array}$ & 5 \\
\hline 7 & cpyl & 39 & 10.8 & 481 & 96 & $\begin{array}{l}\text { L: CTCCTCAAGCTCATGGTGTTT } \\
\text { R: ATTTGACGATGAGGATCGAGAG }\end{array}$ & 3 \\
\hline 8 & fiol-SPAC1F7.09c & 10 & 6.9 & 200 & 81 & $\begin{array}{l}\text { L: CAACAAGTGACAAGCCATGAAT } \\
\text { R: CCTAGGGGTCGAAGTAAGAAGA }\end{array}$ & 2 \\
\hline 9 & tif 471 & 24 & 20.5 & 560 & 85 & $\begin{array}{l}\text { L: GGTGCACTTGATTTGATTTCAG } \\
\text { R: CAACAGCGTATCCAGGAAAAAG }\end{array}$ & 2 \\
\hline 10 & SPAC26F1.13c-SPAC26F1.12c & 21 & 2.5 & 249 & 75 & $\begin{array}{l}\text { L: TTGGATCGTTTGAGCCATATT } \\
\text { R: GGATTAATCGTTGGGAAAATCA }\end{array}$ & 2 \\
\hline 11 & $a p p 1$ & 45 & 12.0 & 585 & 80 & $\begin{array}{l}\text { L: ACCACGGGCTCATTAAGTTGT } \\
\text { R: GGCGCAACCATCAGTCATTA }\end{array}$ & 2 \\
\hline 12 & SPAC11E3.13c-SPAC11E3.14 & 9 & 9.2 & 228 & 67 & $\begin{array}{l}\text { L: TGAAAATGAAAGGAATGCCAAT } \\
\text { R: GTGCACGTTCGCTACATCATA }\end{array}$ & 2 \\
\hline 13 & $r l p 1-\mathrm{SPBC} 1685.13$ & 10 & 10.3 & 182 & 60 & $\begin{array}{l}\text { L: AGGTAGAAGGGATTCGACAGC } \\
\text { R: ACAGGCAGTGGAAGAGAGACA }\end{array}$ & 4 \\
\hline 14 & SPBC36.03c-cys11 & 9 & 13.9 & 298 & 74 & $\begin{array}{l}\text { L: CGATATAGTCGAACATAGCCGTA } \\
\text { R: TGGAGGTGAAAAGGACATAGAA }\end{array}$ & 3 \\
\hline 15 & wis1-SPBC409.08 & 9 & 5.2 & 242 & 76 & $\begin{array}{l}\text { L: GACTACATCGGCAAAAACACC } \\
\text { R: GCAAGCAAATTACACCTGACC }\end{array}$ & 2 \\
\hline 16 & $r p b 1$ & 21 & 25.5 & 599 & 80 & $\begin{array}{l}\text { L: GATATTCCACGTCACCTGCTTA } \\
\text { R: CCAAAAGCATTGTCATCTTCAC }\end{array}$ & 2 \\
\hline 17 & SPBC1711.05 & 54 & 8.7 & 571 & 92 & $\begin{array}{l}\text { L: CATCCGGAAGTGGATCTTCAT } \\
\text { R: CTTTCGGAGTCAGAGGAATCAG }\end{array}$ & 3 \\
\hline 18 & SPBC19C7.10 & 33 & 4.8 & 245 & 77 & $\begin{array}{l}\text { L: GACACCTATCAAGCCTTCTCTTC } \\
\text { R: CGAACTTCTTTTCCAACTTTCC }\end{array}$ & 1 \\
\hline 19 & nep1 & 33 & 3.9 & 244 & 83 & $\begin{array}{l}\text { L: ATCACCACCATCATCATCACA } \\
\text { R: TGGACAGAAGGTAATGGCGTA }\end{array}$ & 2 \\
\hline 20 & sat1-ssn 6 & 9 & 7.2 & 296 & 75 & $\begin{array}{l}\text { L: GGAGGTAGGAGGAACGCTAGAT } \\
\text { R: ATTGGATGTTTAGTTGGCGAGT }\end{array}$ & 6 \\
\hline 21 & SPBC26H8.09c & 33 & 2.6 & 595 & 69 & $\begin{array}{l}\text { L: GCTCTGAATCAATGCGATGTA } \\
\text { R: TTTCGTTTGACACCAACTTTTC }\end{array}$ & 2 \\
\hline 22 & SPCC 330.04c & $21+33$ & $6.1+6$ & 400 & 94,99 & $\begin{array}{l}\text { L: AACATCAAGGATTTCTCGAACTA } \\
\text { R: GCCTCCAGCTTGGTTTCG }\end{array}$ & 3 \\
\hline 23 & sap1-SPCC1672.03c & 14 & 7.7 & 226 & 72 & $\begin{array}{l}\text { L: TCTGGGTGAAAGAATGAGCAAT } \\
\text { R: GACGTTTGCAGTGTTGAAGAGA }\end{array}$ & 3 \\
\hline 24 & ent1 & 48 & 8.4 & 489 & 80 & $\begin{array}{l}\text { L: AAAGGGTTGTTGGACTCAAATG } \\
\text { R: TTTCATCCGTCCTCAAAATACC }\end{array}$ & 1 \\
\hline 25 & SPCC1739.07-SPCC1739.08c & 10 & 10.6 & 189 & 63 & $\begin{array}{l}\text { L: ATGAAAGAGGAGGAAAAGAGGT } \\
\text { R: GAATTGAGGCGAAAGAGAGAGA }\end{array}$ & 2 \\
\hline 26 & $w t f 21-a m t 1$ & 7 & 9.3 & 204 & 66 & $\begin{array}{l}\text { L: ATGAGAAGGAGGCGGTAATTTT } \\
\text { R: TCCACTTCCCTATCTGCTTGTT }\end{array}$ & 2 \\
\hline
\end{tabular}


Table 2. cont.

\begin{tabular}{|c|c|c|c|c|c|c|c|}
\hline No. & Position ${ }^{\star}$ & $\begin{array}{l}\text { Repeat unit } \\
\text { length } \dagger\end{array}$ & Copy no. $\ddagger$ & $\begin{array}{l}\text { Fragment } \\
\text { length } \S\end{array}$ & Match (\%) & Primer sequences & Alleles|l \\
\hline 27 & rpp203 & 3 & 17.7 & 220 & 80 & $\begin{array}{l}\text { L: CTGTCGGTATCGAAAGTGAGT } \\
\text { R: TCGTCAGATTCTTCTTCTTCC }\end{array}$ & 1 \\
\hline 28 & isp4-rpl26 & 5 & 13.2 & 233 & 85 & $\begin{array}{l}\text { L: TTTTATGGAATCAACGTCCAC } \\
\text { R: CCCGCTCCAAGATACTAAAA }\end{array}$ & 1 \\
\hline 30 & sat1-ssn6 & 5 & 11.4 & 230 & 88 & $\begin{array}{l}\text { L: TCGAAACAACATACATCCGTA } \\
\text { R: GCTGCACATAAAGGAAAAAGA }\end{array}$ & 1 \\
\hline 31 & rpp102 & 3 & 24.0 & 176 & 71 & $\begin{array}{l}\text { L: TCTTCATCAGACTCCTCTTCTTC } \\
\text { R: ATCTTTGCTAAGGCTTTGGAG }\end{array}$ & 1 \\
\hline 32 & SPCC757.12 & 6 & 8.3 & 244 & 79 & $\begin{array}{l}\text { L: TCCTTCTACTTCGATGAGTGC } \\
\text { R: ACTACTGTATGCCGTGGAAGT }\end{array}$ & 1 \\
\hline
\end{tabular}

${ }^{\star}$ A single gene/systematic name indicates a coding tandem repeat; two names indicate flanking genes of a non-coding tandem repeat.

$\dagger$ Repeats 27-32 are microsatellites.

$\ddagger$ Copy number in sequenced genome.

§Predicted fragment length (bp).

IINumber of alleles observed in six Sch. pombe isolates (for minisatellite markers 1-26) or 16 genetic strains (for microsatellite markers 27-32).

for rRNA sequencing studies (Kurtzman \& Robnett, 1991, 1998). CBS356, CBS357 and CBS5557 have been the subject of Schizosaccharomyces nuclear DNA reassociation studies (Vaughan Martini, 1991), and CBS356, CBS5557 and CBS5682 (also known as CSIR Y-457 and identical to EF3) of mating configuration studies (Schlake \& Gutz, 1993). Zimmer et al. (1987) analysed mitochondrial genome variation with CBS356, CBS2775 and CBS5682; this last strain has also been the subject of comparative transposon analysis (Levin et al., 1990).

Fig. 1 shows that most ( $81 \%$ ) of the bioinformatically identified minisatellites demonstrated polymorphism within these six Sch. pombe isolates. Ten of the 11 loci within intergenic sequences and 11 out of 15 loci within protein-coding sequences had more than one allele identified (Fig. 1, Table 2): just five markers were uninformative within the six isolates. There is no significant difference in the frequencies of informative loci between the protein-coding and non-coding minisatellites $\left[P\left(\chi^{2}\right)>0.1\right]$; the frequencies of moderately polymorphic loci containing two to three alleles are similar between these sequence types and each contains one highly polymorphic locus with more than four alleles (Fig. 2). As expected, the $972 h^{-}$and 975 $h^{+}$Leupold strains were indistinguishable with all tandem repeat markers (Fig. 1, lanes 1 and 2).

None of the minisatellite markers contained a perfect tandem repeat sequence (Table 2). Overall, the observed degree of polymorphism (number of alleles) was not correlated with internal percentage match for the tandem repeats $\left(r^{2}=0.018, P=0.52\right)$, but there was a significant association for those tandem repeats in protein-coding regions $\left(r^{2}=0.34, P=0.02\right)$. There was no correlation between number of alleles and the percentage of indels in the tandem repeats $\left(r^{2}=0.023, P=0.46\right)$.

\section{Analysis of polymorphic minisatellite alleles}

The data in Fig. 1 demonstrate that six Sch. pombe isolates of different geographical origin can be distinguished by tandem repeat length using minisatellite markers. An unrooted phenetic dendrogram was constructed of the relationships between the $S c h$. pombe isolates, based on a distance matrix of the polymorphic minisatellite alleles. The tree was constructed with the neighbour-joining program of PHYLIP and the input order was randomized to avoid construction bias. The tree obtained (Fig. 3) groups the isolates 972/975 (French) and CBS5557 (Spanish), both of which originate from wine production with grapes. The CBS357 (Jamaican) and CBS2775 (Japanese) isolates were both taken from fermenting molasses: for the Jamaican source this was intended for rum making and originated from cane sugar production. These have been grouped with the isolate from local beer, CBS5682 (South African). The origin of the type strain CBS356 is uncertain but it may derive from the original Lindner strain; Fig. 3 shows that it is clearly distinct from the other five isolates. CBS356 was obtained from the Král Collection, the world's first microbial culture collection, which was established in Prague in 1890, the same year as the shipment of millet beer from East Africa from which Lindner first isolated and described Sch. pombe (Lindner, 1893).

The allele size differences in Fig. 1 are consistent with integer differences in copy numbers of tandem repeats, and to check this PCR products were sequenced from different alleles for two polymorphic loci: tandem repeats in genes $r p b 1$ and 

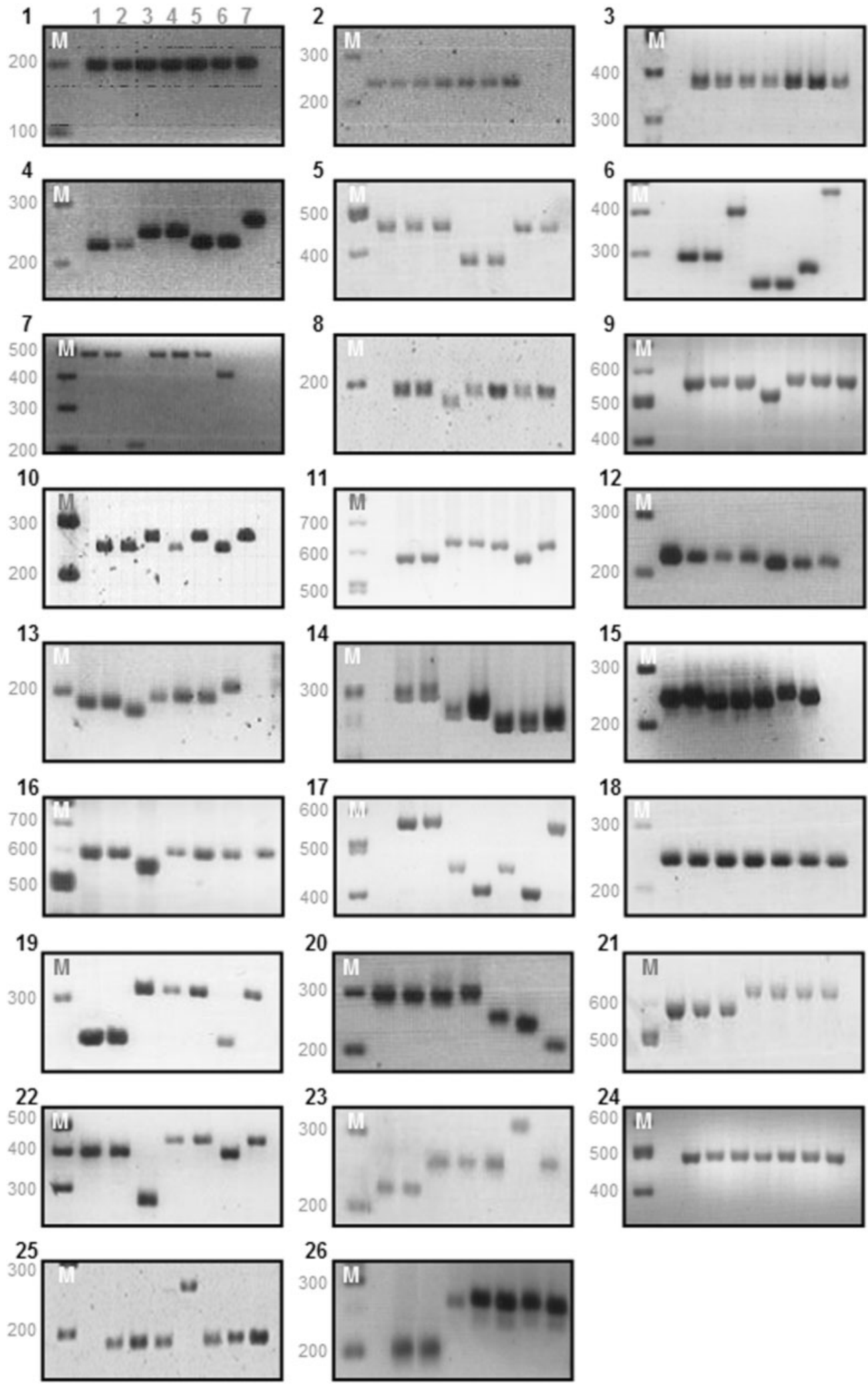

Fig. 1. Genetic fingerprinting of Sch. pombe isolates. Images 1-26 show PCR amplification products of the respective minisatellite tandem repeats $1-26$ in Table 2 from Sch. pombe strains $972 h^{-}$(lane1), $975 h^{+}$(lane 2), CBS356 (lane 3), CBS357 (lane 4), CBS2775 (lane 5), CBS5557 (lane 6), and CBS5682 (lane 7). The marker lane (M) contains 100 bp ladder. 


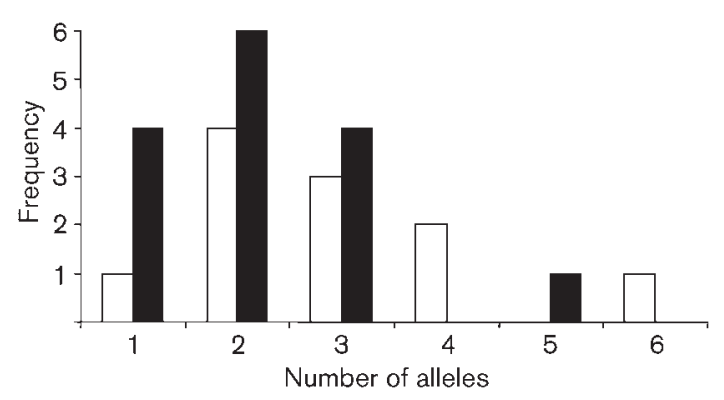

Fig. 2. Number of alleles identified in six Sch. pombe isolates for minisatellite loci within 15 protein-coding sequences (black bars) and 11 intergenic sequences (white bars).

SPCC330.04c. For both loci the sequences corresponded with the allele sizes observed by gel electrophoresis and confirmed that the alleles differ by integer numbers of repeat units. The rpb1 gene in Sch. pombe isolate CBS356 contains only 23 copies of the $21 \mathrm{bp}$ tandem repeat as compared with 25 copies in the Osterwalder isolate (see supplementary Fig. S1, available with the online version of this paper). The encoded heptapeptide repeat is in the essential C-terminal domain (CTD) of the large subunit of RNA polymerase II; this repeat is known to vary in copy number between species (Stiller \& Hall, 2002) but to our knowledge this is the first report of a CTD repeat copy number variation between naturally occurring strains of Sch. pombe. A more complex pattern was observed in the three alleles of the SPCC 330.04c gene, where the Osterwalder isolate has six repeats of $21 \mathrm{bp}$ followed by six repeats of a $33 \mathrm{bp}$ unit, the CBS356 isolate has three copies of the $21 \mathrm{bp}$ unit and only four of the $33 \mathrm{bp}$ unit whereas CBS2775 has five repeats of the $21 \mathrm{bp}$ unit and eight of the 33 bp unit (see supplementary Fig. S2). This interesting pattern of switching from a repeat unit size of 21 bp to a $33 \mathrm{bp}$ unit is discussed below, but again the sequencing confirmed that the alleles all differed by integer numbers of repeat units. At both loci mutations seem to affect the middle of the tandemly repeated region and this is most evident in the multiple alignment of the SPCC330.04c gene sequences. Within the 21 bp repeated sequence isolate CBS2775 has lost the third unit and CBS356 has lost the third, fourth and fifth units but the first, second and sixth units remain unchanged compared with the Osterwalder strain. Within the $33 \mathrm{bp}$ repeated sequence CBS356 has lost the fourth and fifth repeat units, and isolate CBS2775 has gained two units after repeat five, compared with the Osterwalder isolate. At both loci the tandem repeat sequences for the $972 h^{-}$or $975 h^{+}$strains were identical to the published sequence for the $972 h^{-}$strain (Wood et al., 2002).

\section{Minisatellite and microsatellite polymorphisms in genetic strains of Sch. pombe}

Virtually all strains used for Sch. pombe genetics are derived from homothallic and heterothallic strains obtained by Leupold (1950) from the Osterwalder (1924) isolate, and are assumed to have isogenic backgrounds. The high level of

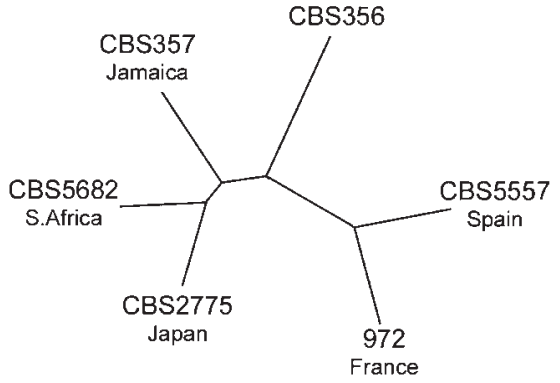

Fig. 3. Unrooted phenetic tree illustrating relationships between Sch. pombe isolates of different geographical origin constructed by the neighbour-joining method based on a distance matrix of polymorphic minisatellite alleles. CBS356 is the type strain of Sch. pombe Lindner; 972 is an $h^{-}$genetic strain derived from the Osterwalder isolate formerly named Sch. liquefaciens; CBS357 was formerly Sch. mellacei; CBS2775 was formerly Sch. pombe var. ogasawaraensis; CBS5557 was formerly Sch. malidevorans.

variation seen with tandem repeat markers provides the opportunity to test this assumption. We therefore extended the tandem repeat marker analysis to a selection of genetic strains obtained from a diversity of laboratories in the Sch. pombe community (Table 1), but all ultimately derived from Leupold strains. The strains included mutants defective in DNA replication ( $c d c 23, h s k 1, n d a 4)$, cell cycle checkpoints (cut5, rad3), mitosis (cdc25, nda3) and recombination (swi5). In addition to the 26 minisatellite markers described above a further six tandem repeats were also selected with repeat unit sizes of $\leqslant 6 \mathrm{bp}$, i.e. microsatellite markers (Table 2, numbers 27-32). All 32 pairs of primers were applied to each of 16 genetic strains.

No tandem repeat differences were detected between any of the genetic strains with 30 of the 32 markers (data not shown). The two exceptional polymorphic loci were the minisatellite in the tif471 gene, for which one strain exhibited a shorter allele (supplementary Fig. S3), and the microsatellite marker in the med15 gene, which gave five alleles in genetic strains (Fig. 4a). The tif471 gene encodes the translation factor eIF4G scaffold protein that mediates the binding of mRNA and associated proteins to the $43 \mathrm{~S}$ ribosomal preinitiation complex, overexpression of which causes aberrant $S c h$. pombe cell morphology (HashemzadehBonehi et al., 2003). Sequencing of both of the tif 471 alleles revealed that three 24 bp repeat units had been lost from the SJA302 strain in comparison to the $972 h^{-}$strain (supplementary Fig. S3).

The five alleles of med 15 were sequenced and all were found to differ from the 32 repeat units predicted by the published sequence (Fig. 4). The shortest repeated region is found in laboratory strain SJA273, with just over half of the published number of repeated units (17 units) and the longest in strain SJA272 with 40 repeated units; these are consistent with the estimates from gel electrophoresis. This hypervariable 
(a)

$\begin{array}{lllllllllllllllll}M & 1 & 2 & 3 & 4 & 5 & 6 & 7 & 8 & 9 & 10 & 11 & 12 & 13 & 14 & 15 & 16\end{array}$

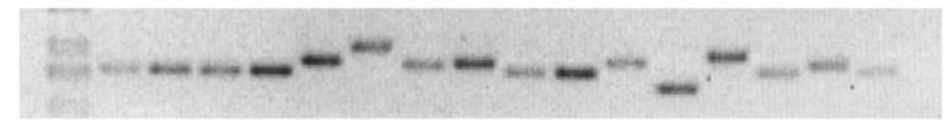

(b)

published ATTCAAAAATTTCGTTCATTGCAGCATATGCAGCAACAACAGCAGCAACAACAACAACAA 975 ATTCAAAAATTTCGTTCATTGCAGCATATGCAGCAACAACAGCAGCAACAACAACAACAA SJA273 ATTCAAAA-TTTCGTTCATTGCAGCATATGCAGCAACAACAGCAGCAACAACAACAACAA SJA302 ATTCAAAAATTTCGTTCATTGNNTTATATGCAGCAACAACAGCAGCAACAACAACAACAA SJA216 ATTCAAAAATTTCGTTCATTGCAGCATATGCAGCAACAACAGCAGCAACAACAACAACAA SJA122 ATTCAAAAATTTCGTTCATTGCAGCATATGCAGCAACAACAGCAGCAACAACAACAACAA SJA120 ATTCAAAAATTTCGTTCATTGCAGCATATGCAGCAACAACAGCAGCAACAACAACAACAA SJA272 ATTCAAAAATTTCGTTCATTGCNTTATATGCAGCAACAACAGCAGCAACAACAACAACAA

published CAACAACAACAACAACAACAACAACAACAACAACAACAACAACAACAACAACAACAACAA 975

SJA 273

SJA 302

SJA216

SJA122

SJA120

SJA272

published

975

SJA273

SJA 302

SJA2 16

SJA122

SJA120

SJA272

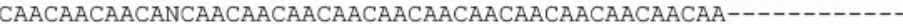

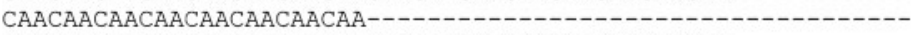

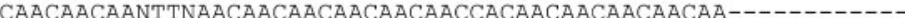
CAACAACAACAACAACAACAACAACAACAACAACAACAACAACAACAACAACAACAACAA CAACAACAACAACAACAACAACAACAACAACAACAACAACAACAACAACAACAACAACAA CAACAACAACAACAACAACAACAACAACAACAACAACAACAACAACAACAACAACAACAA CAACAACAACNNCAACAACAACAACAACAACAACAACAACAACAACAACAACAACAACAA

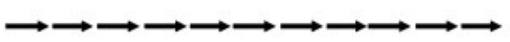

CAACAACAA--------CAACAACAA 作 CAACAN----------------------AAGNAAGCTCCGCATNATGCTTTTTTT

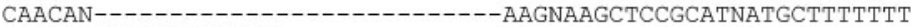
CAACAACAACAACAACAN------------AAGNANGCTCCGNATGATGCTTTTTTT CAACAACAACAACAACAACAACAACAACAACAGAAGNANGCTCCGNANGATGCTTTTTTT
Fig. 4. Polymorphism of the med15 microsatellite tandem repeat locus in Sch. pombe laboratory strains. (a) PCR amplification products of the tandem repeat locus within the protein-coding sequence of med15 analysed by agarose gel electrophoresis for laboratory strains: lane 1, $972 h^{-}$; lane 2, $975 h^{+}$; lane 3, SJA148; lane 4, SJA167; lane 5, SJA122; lane 6, SJA272; lane 7, SJA237; lane 8, SJA175; lane 9, SJA277; lane 10, SJA244; lane 11, SJA216; lane 12, SJA273; lane 13, SJA120; lane 14, SJA238; lane 15, SJA300; lane 16, SJA302. (b) Alignment of the published 972 $h^{-}$sequence (Wood et al., 2002) with sequenced PCR products for strains 975 $h^{+}$, SJA273, SJA302, SJA216, SJA122, SJA120 and SJA272. The $3 \mathrm{bp}$ repeat units are indicated by arrows. tandem repeat encodes a homopolymeric stretch of glutamine residues within the predicted Med15 protein, which is an orthologue of Gal11, a component of the Mediator complex which forms part of the RNA polymerase II holoenzyme (The Wellcome Trust Sanger Institute; www.genedb.org).

\section{DISCUSSION}

The popularity of Sch. pombe as a model organism has concentrated studies of this species on genetic strains derived by Leupold (1950) from a single wild-type isolate (Osterwalder, 1924). Our data open the door to molecular analysis of natural variation in this fission yeast species by providing 21 minisatellite markers for genetic fingerprinting of different isolates of Sch. pombe, and provide evidence of some variation between genetic strains. The minisatellite allele differences can be detected using agarose gels, without recourse to sequencing; therefore the markers can be readily used for distinguishing between strains of Sch. pombe. We attempted to extend the analysis to other members of the genus Schizosaccharomyces by using the same primers against Sch. japonicus and Sch. octosporus DNA. However, no reproducible PCR products were obtained for Sch. octosporus and only faint bands were visible for Sch. japonicus when using very low annealing temperatures, which did not match any of the alleles identified in the Sch. pombe isolates (data not shown). Attempts by Ingavale et al.
(1998) to amplify the two tandem repeats in the cpyl gene of Sch. pombe in Sch. japonicus and Sch. octosporus similarly failed to give detectable products. This failure of Sch. pombe primers to anneal to DNA in the other two Schizosaccharomyces species indicates that there is significant sequence divergence between the three species within this genus, and is consistent with the conclusion from rRNA sequencing that the three recognized fission yeast species are not closely related (Kurtzman \& Robnett, 1991, 1998).

Ingavale et al. (1998) analysed tandem repeats in the vacuolar carboxypeptidase Y ( $c p y 1)$ gene of two isolates of Sch. pombe and found just one difference between the Osterwalder isolate and the Rankine \& Fornachon isolate CBS5557 formerly known as Sch. malidevorans. We have confirmed these data and extended them to four more Sch. pombe isolates, discovering three and five distinct alleles for the respective RS I and RS II markers in this gene (Fig. 1: gels 7 and 6). In addition we have identified a further 19 minisatellite markers at other loci which are informative for fingerprinting strains of Sch. pombe. Only five of the tandem repeats we tested were uninformative (Fig. 2), although it is possible that some of these may show polymorphisms if analysed in other Sch. pombe isolates.

All of the minisatellite polymorphic markers were imperfect tandemly repeated sequences and overall there was little correlation between internal conservation of sequence and the number of polymorphic alleles. This supports the 
conclusion of Denoeud et al. (2003) that measurements of overall internal conservation, used as a polymorphism predictor for microsatellites, are not applicable to minisatellites, presumably owing to the greater complexity of their mutation processes. Our sequence data do indicate that polymorphisms occur at particular internal sites in the tandem repeats where neighbouring units share a high degree of sequence conservation, rather than in the terminal repeat units. However, these internal sites should not be looked at in isolation because they form part of larger repetitive elements, which are important to give a complete picture, particularly in evolutionary studies. Five of our Sch. pombe minisatellites exhibited polymorphism despite having less than $70 \%$ sequence match between individual repeat units. This demonstrates the great importance of considering imperfect as well as perfect tandem repeats in any study related to expansion and contraction of such sequences.

Surprisingly, there was no significant difference in the frequency of informative loci between protein-coding and non-coding tandem repeats. This shows that length variations can be tolerated in many Sch. pombe proteins. Gene ontology (GO) analysis of the ten polymorphic proteins reveals a wide diversity of biological process and molecular function, but all of the proteins are predicted to be intracellular and four are nuclear. These are therefore very different proteins from the set of serine/threoninerich repeat-containing cell wall proteins identified in Sac. cerevisiae by Verstrepen et al. (2005) and Richard \& Dujon (2006). All the Sch. pombe polymorphic tandem repeats are predicted to be in regions with no discernible 3D structure, and for only the repeats in SPAC16E8.01 ( $\beta$ sheet/coil), SPCC330.04c and Tif471 ( $\alpha$-helices) are rigid secondary structures predicted. It therefore appears that these length variations mostly correspond to structurally flexible regions of the encoded proteins. The high mutation rates could increase the evolutionary effects of variation on cellular regulatory processes (Tompa, 2003). Our identification of polymorphic protein repeats in Sch. pombe can allow further investigation of such elements and comparison to those identified in Sac. cerevisiae and other eukaryotes.

The SPCC330.04c protein contains a complex tandem repeat encoded by $21 \mathrm{bp}$ and $33 \mathrm{bp}$ repeat units (supplementary Fig. S2) which is predicted to form an $\alpha$-helical secondary structure, part of which is markedly amphipathic. The repeat units of $21 \mathrm{bp}$ and $33 \mathrm{bp}$ correspond to two and three $\alpha$-helical turns respectively and allele sizes are consistent with integer differences in the tandem repeats; therefore this protein could vary between strains simply by differences in the length of the $\alpha$-helix, not in the relative orientation of its residues or adjoining tertiary structure. This 'integer-helix' model can also explain the pattern of switching from $21 \mathrm{bp}$ to $33 \mathrm{bp}$ repeat units within the one tandem repeat.

Genetic strains of Sch. pombe, unlike those of Sac. cerevisiae, are all derived from one original isolate and are therefore assumed to be isogenic. The availability of DNA fingerprinting markers has allowed this hypothesis to be tested. Reassuringly for the Sch. pombe community, we found no differences between 16 genetic strains for 30 out of 32 tandem repeats we tested, including six microsatellites. The exceptions were the repeat in the tif 471 gene which in a single genetic strain exhibited the shorter allele also observed in the CBS357 isolate, and the trinucleotide repeat in med 15 which appears to be hypervariable. It is possible that the presence of checkpoint or cell cycle mutations may have influenced the probability of genomic changes, although the DNA replication hskl mutant, which has a length mutation at the tif 471 locus, appears to be in the ancestral state at the med15 locus. In the med15 gene five different alleles were detected in the otherwise essentially isogenic 16 genetic strains examined. The reason for the hypervariability of this microsatellite in a gene encoding a predicted RNA polymerase II holoenzyme component remains to be determined. One possibility is that it might lie close to a meiotic recombination hotspot; however, it is $>300 \mathrm{~kb}$ from the nearest of the ten meiotic hotspots detected by Steiner \& Smith (2005). Testing of this possibility awaits full genomic mapping of meiotic hotspots in Sch. pombe. The principal mechanism for expansions and contractions of microsatellites is DNA replication slippage (Ellegren, 2004); therefore this is a more likely explanation for variation at this locus. The finding of an unstable microsatellite in Sch. pombe affords the possibility to examine not just the mechanism underlying mutation, but also the particular properties of this locus that confer hypervariability, thereby elucidating mechanisms by which eukaryotic cells normally ensure genome stability at other microsatellite loci.

Few molecular studies have been carried out on natural wild-type variants of Sch. pombe. The chromosomal locations and total numbers of Tf1 and Tf2 retrotransposons differ greatly in various isolates of Sch. pombe (Levin et al., 1990), as might be expected, although the sequences appear to be highly conserved (Hoff et al., 1998). Size differences have been reported in the mitochondrial genomes of different Sch. pombe isolates due to the presence or absence of introns (Zimmer et al., 1987). On the other hand, Takahashi et al. (1992) found that centromeres are highly conserved between natural variants of Sch. pombe. Some strains would particularly merit further study, for example Sch. pombe NCYC 132, which was extensively used for early cell cycle investigations (Mitchison, 1989) and has recently been studied in relation to hyphal growth (Amoah-Buahin et al., 2005), and Sch. kambucha, whose mating-type locus has been sequenced and shows around $2 \%$ difference from standard strains (Singh \& Klar, 2003). Many scores of wildtype strains of Sch. pombe have been isolated, from all inhabited continents of the world and from many types of substrate. The markers described in this study provide a route into population biology and will permit extensive studies of the natural variation of this important model species. 


\section{ACKNOWLEDGEMENTS}

We thank Matthew Redden, Kirstie Affleck, Charlotte Cymbalista and Daniel Harrison for valuable contributions to the project, and Antony Carr, Richard Egel, Susan Forsburg, Paul Nurse, Henning Schmidt, Pierre Thuriaux and Mitsuhiro Yanagida for Sch. pombe strains. A.M.P. was funded by a studentship from the Biotechnology and Biological Sciences Research Council (BBSRC) of the UK and Kirstie Affleck by a Genetics Society vacation studentship.

\section{REFERENCES}

Amoah-Buahin, E., Bone, N. \& Armstrong, J. (2005). Hyphal growth in the fission yeast Schizosaccharomyces pombe. Eukarot Cell 4, 1287-1297.

Benson, G. (1999). Tandem repeats finder: a program to analyze DNA sequences. Nucleic Acids Res 27, 573-580.

Boby, T., Patch, A.-M. \& Aves, S. J. (2005). TRbase: a database relating tandem repeats to disease genes for the human genome. Bioinformatics 21, 811-816.

Bowen, S. \& Wheals, A. E. (2006). Ser/Thr-rich domains are associated with genetic variation and morphogenesis in Saccharomyces cerevisiae. Yeast 23, 633-640.

Calafell, F., Shuster, A., Speed, W. C., Kidd, J. R. \& Kidd, K. K. (1998). Short tandem repeat polymorphism evolution in humans. Eur J Hum Genet 6, 38-49.

Chambers, G. K. \& MacAvoy, E. S. (2000). Microsatellites: consensus and controversy. Comp Biochem Physiol B Biochem Mol Biol 126, 455-476.

Debrauwère, H., Gendrel, C. G., Lechat, S. \& Dutreix, M. (1997). Differences and similarities between various tandem repeat sequences: minisatellites and microsatellites. Biochimie 79, 577-586.

Denoeud, F., Vergnaud, G. \& Benson, G. (2003). Predicting human minisatellite polymorphism. Genome Res 13, 856-867.

Egel, R. (2004). The Molecular Biology of Schizosaccharomyces pombe: Genetics, Genomics and Beyond. Berlin: Springer.

Ellegren, H. (2004). Microsatellites: simple sequences with complex evolution. Nat Rev Genet 5, 435-445.

Felsenstein, J. (2005). PHYLIP - Phylogeny Inference Package, version 3.6. Distributed by the author. University of Washington, Seattle, USA.

Fondon, J. W., III \& Garner, H. R. (2004). Molecular origins of rapid and continuous morphological evolution. Proc Natl Acad Sci U S A 101, 18058-18063.

Forsburg, S. L. (1999). The best yeast? Trends Genet 15, 340-344.

Forsburg, S. L. \& Rhind, N. (2006). Basic methods for fission yeast. Yeast 23, 173-183.

Hashemzadeh-Bonehi, L., Curtis, P. S., Morley, S. J., Thorpe, J. R. \& Pain, V. M. (2003). Overproduction of a conserved domain of fission yeast and mammalian translation initiation factor eIF4G causes aberrant cell morphology and results in disruption of the localization of F-actin and the organization of microtubules. Genes Cells $\mathbf{8}$, 163-178.

Heckman, D. S., Geiser, D. M., Eidell, B. R., Stauffer, R. L., Kardos, N. L. \& Hedges, S. B. (2001). Molecular evidence for the early colonization of land by fungi and plants. Science 293, 1129-1133.

Hoff, E. F., Levin, H. L. \& Boeke, J. D. (1998). Schizosaccharomyces pombe retrotransposon Tf2 mobilizes primarily through homologous cDNA recombination. Mol Cell Biol 18, 6839-6852.

Ingavale, S. S., Kaur, R., Aggarwal, P. \& Bachhawat, A. K. (1998). A minisatellite sequence within the propeptide region of the vacuolar carboxypeptidase Y gene of Schizosaccharomyces pombe. J Bacteriol 180, 3727-3729.

Kashi, Y., King, D. \& Soller, M. (1997). Simple sequence repeats as a source of quantitative genetic variation. Trends Genet 13, 74-78.

Katti, M. V., Ranjekar, P. K. \& Gupta, V. S. (2001). Differential distribution of simple sequence repeats in eukaryotic genome sequences. Mol Biol Evol 18, 1161-1167.

Kellis, M., Patterson, N., Endrizzi, M., Birren, B. \& Lander, E. S. (2003). Sequencing and comparison of yeast species to identify genes and regulatory elements. Nature 423, 241-254.

Kurtzman, C. P. \& Robnett, C. J. (1991). Phylogenetic relationships among species of Saccharomyces, Schizosaccharomyces, Debaryomyces and Schwanniomyces determined from partial ribosomal RNA sequences. Yeast 7, 61-72.

Kurtzman, C. P. \& Robnett, C. J. (1998). Identification and phylogeny of ascomycetous yeasts from analysis of nuclear large subunit (26S) ribosomal DNA partial sequences. Antonie van Leeuwenhoek 73, 331-371.

Leupold, U. (1950). Die Vererbung von Homothallie und Heterothallie bei Schizosaccharomyces pombe. C R Lab Carsberg Sér Physiol 24, 381-480.

Levin, H. L., Weaver, D. C. \& Boeke, J. D. (1990). Two related families of retrotransposons from Schizosaccharomyces pombe. Mol Cell Biol 10, 6791-6798.

Lindner, P. (1893). Schizosaccharomyces pombe n. sp., ein neuer Gährungserreger. Wochenschr Brauerei 10, 1298-1300.

Marguerat, S., Jensen, T. S., de Lichtenberg, U., Wilhelm, B. T., Jensen, L. J. \& Bähler, J. (2006). The more the merrier: comparative analysis of microarray studies on cell cycle-regulated genes in fission yeast. Yeast 23, 261-277.

McGuffin, L. J., Bryson, K. \& Jones, D. T. (2000). The PSIPRED protein structure prediction server. Bioinformatics 16, 404-405.

Mitas, M. (1997). Trinucleotide repeats associated with human disease. Nucleic Acids Res 25, 2245-2254.

Mitchison, J. M. (1989). Cell cycle growth and periodicities. In Molecular Biology of the Fission Yeast, pp. 205-242. Edited by A. Nasim, P. Young \& B. F. Johnson. San Diego: Academic Press.

Moreno, S., Klar, A. \& Nurse, P. (1991). Molecular genetic analysis of fission yeast Schizosaccharomyces pombe. Methods Enzymol 194, 795-823.

Osterwalder, A. (1924). Schizosaccharomyces liquefaciens n. sp., eine gegen freie schweflige Säure widerstandsfähige Gärhefe. Mitt Gebiete Lebensmittelunters Hyg 15, 5-28.

Peng, K., Radivojac, P., Vucetic, S., Dunker, A. K. \& Obradovic, Z. (2006). Length-dependent prediction of protein intrinsic disorder. BMC Bioinformatics 7, 208.

Richard, G. F. \& Dujon, B. (2006). Molecular evolution of minisatellites in hemiascomycetous yeasts. Mol Biol Evol 23, 189-202.

Richard, G. F. \& Pâques, F. (2000). Mini- and microsatellite expansions: the recombination connection. EMBO Rep $\mathbf{1}$, 122-126.

Rost, B., Yachdav, G. \& Liu, J. (2003). The PredictProtein Server. Nucleic Acids Res 32 (Web Server issue), W321-W326.

Rozen, S. \& Skaletsky, H. J. (2000). Primer3 on the WWW for general users and for biologist programmers. In Bioinformatics Methods and Protocols: Methods in Molecular Biology, pp. 365-386. Edited by S. Krawetz \& S. Misener. Totowa, NJ: Humana Press.

Schlake, T. \& Gutz, H. (1993). Mating configurations in Schizosaccharomyces pombe strains of different geographical origins. Curr Genet 23, 108-114. 
Singh, G. \& Klar, A. J. (2003). DNA sequence of the mat2,3 region of Schizosaccharomyces kambucha shares high homology with the corresponding sequence from Sz. pombe. Yeast 20, 1273-1278.

Sipiczki, M. (2000). Where does fission yeast sit on the tree of life? Genome Biol 1, reviews 1011.1-1011.4.

Steiner, W. W. \& Smith, G. R. (2005). Natural meiotic recombination hot spots in the Schizosaccharomyces pombe genome successfully predicted from the simple sequence motif M26. Mol Cell Biol 25, 9054-9062.

Stiller, J. W. \& Hall, B. D. (2002). Evolution of the RNA polymerase II C-terminal domain. Proc Natl Acad Sci U S A 99, 6091-6096.

Strand, M., Prolla, T. A., Liskay, R. M. \& Petes, T. D. (1993). Destabilization of tracts of simple repetitive DNA in yeast by mutations affecting DNA mismatch repair. Nature 365, 274-276.

Sunnerhagen, P. (2002). Prospects for functional genomics in Schizosaccharomyces pombe. Curr Genet 42, 73-84.

Sutherland, G. R. \& Richards, R. I. (1995). Simple tandem DNA repeats and human genetic disease. Proc Natl Acad Sci U S A 92 3636-3641.

Takahashi, K., Murakami, S., Chikashige, Y., Funabiki, H., Niwa, O. \& Yanagida, M. (1992). A low copy number central sequence with strict symmetry and unusual chromatin structure in fission yeast centromere. Mol Biol Cell 3, 819-835.

Thompson, J. D., Higgins, D. G. \& Gibson, T. J. (1994). CLUSTALW: improving the sensitivity of progressive multiple sequence alignment through sequence weighting, position-specific gap penalties and weight matrix choice. Nucleic Acids Res 22, 4673-4680.
Tompa, P. (2003). Intrinsically unstructured proteins evolve by repeat expansion. Bioessays 25, 847-855.

Tóth, G., Gaspari, Z. \& Jurka, J. (2000). Microsatellites in different eukaryotic genomes: survey and analysis. Genome Res 10, 967-981.

Vaughan Martini, A. (1991). Evaluation of phylogenetic relationships among fission yeast by nDNA/nDNA reassociation and conventional taxonomic criteria. Yeast 7, 73-78.

Vergnaud, G. \& Denoeud, F. (2000). Minisatellites: mutability and genome architecture. Genome Res 10, 899-907.

Verstrepen, K. J., Jansen, A., Lewitter, F. \& Fink, G. R. (2005). Intragenic tandem repeats generate functional variability. Nat Genet 37, 986-990.

Viguera, E., Canceill, D. \& Ehrlich, S. D. (2001). Replication slippage involves DNA polymerase pausing and dissociation. EMBO J 20, 2587-2595.

Ward, J. J., Sodhi, J. S., McGuffin, L. J., Buxton, B. F. \& Jones, D. T. (2004). Prediction and functional analysis of native disorder in proteins from the three kingdoms of life. J Mol Biol 337, 635-645.

Wood, V., Gwilliam, R., Rajandream, M. A., Lyne, M., Lyne, R., Stewart, A., Sgouros, J., Peat, N., Hayles, J. \& other authors (2002). The genome sequence of Schizosaccharomyces pombe. Nature 415, 871-880.

Zimmer, M., Welser, F., Oraler, G. \& Wolf, K. (1987). Distribution of mitochondrial introns in the species Schizosaccharomyces pombe and the origin of the group II intron in the gene encoding apocytochrome b. Curr Genet 12, 329-336.

Edited by: H. A. B. Wösten 